\title{
Application of R Software in the Experiment Teaching of Medical Advanced Mathematics
}

\author{
Jun Zhao ${ }^{1, ~ a ~ a n d ~ L i n ~ Y a n g ~}{ }^{1, b^{*}}$ \\ ${ }^{1}$ Institute of Medicine and Nursing, Hubei University of Medicine, Shiyan, China \\ a427511@qq.com, b5810030@ qq.com \\ *The corresponding author
}

Keywords: Advanced mathematics; Mathematical experiment; Reform in education; R software

\begin{abstract}
Experimental teaching is an important part of Medical Advanced Mathematics. The software R, which is widely used, is an open source mathematical computing environment. It has powerful functions and plenty of packages. After analyzing the practical problems in college mathematics teaching, this paper explored the feasibility and superiority of the application of $\mathrm{R}$ software assisting mathematics teaching in medical colleges by combining with the $\mathrm{R}$ software in mathematics teaching in specific application examples. This can provide some references in the teaching reform of college mathematics curriculum.
\end{abstract}

\section{$\mathrm{R}$ 软件在《医用高等数学》实验教学中的应用}

\author{
赵军 $1^{1, \mathrm{a}}$, 杨琳 $1^{1, \mathrm{~b}}$ \\ 1. 湖北医药学院药护学院, 中国 湖北十堰 442000 \\ a427511@qq.com, ${ }^{b} 5810030 @ q q . c o m$
}

摘要: 实验教学是《医用高等数学》课程教学的重要组成部分; $\mathrm{R}$ 软件应用非常广泛, 它是 一种开源的数学计算环境, 拥有强大的功能和丰富的扩展包。本文分析了医学数学实验教学 中的现实问题, 结合 $\mathrm{R}$ 软件在数学基础实验教学中的具体应用实例, 探讨在医学院校应用 $\mathrm{R}$ 软件开展数学实验课的可行性和优越性, 为医学院校的数学课程教学改革提供一些参考。

关键词: 高等数学; 数学实验; 教学改革; $R$ 软件

\section{1. 引言}

《医用高等数学》是高等医学院校一门重要的必修基础课程, 对学生思维素质的培养和后继 课程的学习起着重要的作用。但是, 由于和医学专业的直接联系不紧密, 在医学院校开设高 等数学课程的必要性一直受到许多学生的质疑 [1]。另一方面, 高等数学的抽象性也极易使学 生产生畏难情绪, 失去学习兴趣。如何提高医学生学习高等数学的积极性, 是摆在每一位医 学院校高等数学教师面前的首要问题。数学实验是数学教学的重要组成部分, 它着重训练学 生运用数学知识解决实际问题的技巧和技能。在数学实验中, 学生通过亲身的实践能从大量 的实际问题中体会到高等数学知识的广泛应用性，从而激发学习兴趣。自由软件 $\mathrm{R}$ 设计开发 的初衷是用于统计计算, 但它也是一套完整的数据处理、计算和制图软件系统 [2]。国外众多 大学都将 $R$ 软件作为教学工具。国内将 $R$ 软件运用于教学的案例直到近些年才有报道 [3-6]。 本文以若干实例验证了其完全可以满足《医用高等数学》实验教学的需求。另外, 数学实验 课中 $\mathrm{R}$ 软件的学习实践, 也会为学生随后学习《医学统计学》课程打下良好的基础。通过统 一实验课软件工具, 两门课程可以实现自然衔接, 大大提高教学效率。 


\section{2. 医学数学实验教学}

数学实验课在理工科专业应用较早较广泛, 而医学院校学生的数学基础和要求与之不同, 所 以通常的医学数学实验教学内容相应简化。《医用高等数学》理论课程包括函数与极限、一元 函数微积分、多元函数微积分、微分方程、概率论基础等内容 [7]。数学实验的基础部分主要 围绕医用高等数学的基本内容。而综合实验部分可以让学生运用已掌握的数学知识和实验知 识, 完整的进行数学建模实验。

在高等数学实验教学中, 应用最广泛的工具无疑是 MATLAB 软件。然而这是一款商业软件, 售价较昂贵 (标准版为 15000 元人民币, 教育版为 3000 元人民币)。在我国日益重视知识产 权的大环境下, 教学单位若想将其应用于日常教学, 必须有足够的经费支持。对于学生来说, 日常的下载安装和自主学习也会遇到障碍。实际上, $\mathrm{R}$ 软件是我们的另外一个好的选择。

\section{3. $\mathrm{R}$ 软件简介}

$\mathrm{R}$ 是一种在科研领域尤其是在数据统计分析中应用非常广泛的软件。其功能包括数据存储、 系统处理、数组运算等, 其向量、矩阵运算方面功能尤其强大。 $\mathrm{R}$ 软件是免费、开源的。与 商业软件动轩上万的购置费及昂贵的维护升级费用相比, 免费的 $\mathrm{R}$ 软件数据分析和建模能力 毫不逊色, 而且比封装、集成的商业软件更具有灵活性。 $\mathrm{R}$ 软件功能广泛, 仅 $\mathrm{R}$ 软件主网站 (https://cran.r-project.org) 就提供了超过七千个程序包, 涵盖基础统计学、社会学、经济管 理、金融、生态、生物信息等多个方面。各种包都是可编辑和重新编译的, 使用者可根据自 身需求, 量身定做独特的程序包。 $\mathrm{R}$ 还提供了各种数学计算的函数, 使用者能灵活机动的进 行数值计算和模拟, 甚至创造出符合需要的新的计算方法。所以与其说 $\mathrm{R}$ 是一种统计软件, 不如说 $\mathrm{R}$ 是一种数学计算的环境 $[8]$ 。 $\mathrm{R}$ 是解释性语言 $\mathrm{S}$ 的一种实现, 简单易学, 作为非计算 机专业的医学生能够轻松上手, 一天之内就能掌握其基本命令, 一周内就能使用其进行基本 的运算和数据分析。

\section{4. $\mathrm{R}$ 软件在数学实验中的应用实例}

4.1. 函数图像演示

函数图像可以直观形象地表示函数, 帮助学生理解函数的性质（如单调性、极值、凹凸性以 及确定积分限等)。

实例 1 . 绘制正弦函数图像

$>$ curve ( $\sin ,-3,3$, main $=$ "正弦函数")

实例 2. 绘制标准正态分布的密度函数曲线

> curve(dnorm, -3.5, 3.5, main="标准正态曲线")

实例 3. 绘制自定义的 Logistic 生长曲线

$>\mathrm{f}<-$ function $(\mathrm{x}) \quad \mathrm{k} /\left(1+\mathrm{a} * \exp \left(-\mathrm{b}^{*} \mathrm{x}\right)\right) \quad \#$ 定义函数

$>\mathrm{a}<-10 ; \mathrm{b}<-8 ; \mathrm{k}<-2 \quad$ \# 给参数赋值

$>$ curve(f, main="Logistic 生长曲线") \# 画函数图像

上述 $\mathrm{R}$ 命令的输出见图 1。由上面的实例可以看出, 仅仅一两条命令, $\mathrm{R}$ 就能完成各种函数 图像的绘制。 

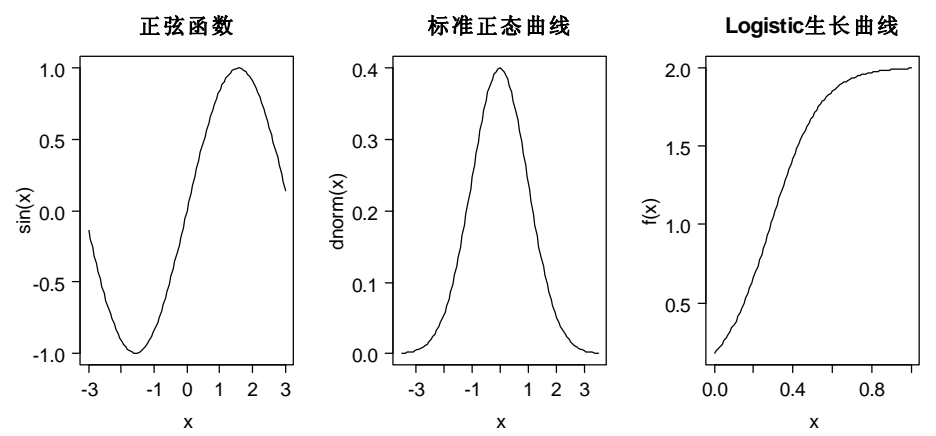

图 1

\section{2. 求方程的根}

数学中很多问题最后都归结为方程的求根问题, 如极值问题。用 $\mathrm{R}$ 软件求方程在指定区间内 的所有根, 需要加载 “rootSolve” 包。

实例 1 . 求方程 $x^{5}-3 x+1=0$ 在区间 $(0,2)$ 内的所有实根

$>$ library(rootSolve) \# 加载 rootSolve 包

$>\mathrm{g}<-\operatorname{function}(\mathrm{x}) \quad \mathrm{x}^{\wedge} 5-3^{*} \mathrm{x}+1 \quad$ \# 定义函数

$>$ uniroot.all $(\mathrm{g}, \mathrm{c}(0,2))$ \#求函数在指定区间的所有零点

上面代码的输出结果为 $0.3347438,1.2146459$, 即为方程在给定区间内的两个实根。

4. 3. 求函数的导数或偏导数

实例 1 . 求函数 $\mathrm{y}=\mathrm{x}^{2}-\sin \mathrm{x}$ 在点 $\mathrm{x}=2$ 处的导数

$>\mathrm{f} 1<-\operatorname{expression}\left(\mathrm{x}^{\wedge} 2-\sin (\mathrm{x})\right) \quad$ \# 定义函数表达式

$>\mathrm{dx}<-\operatorname{deriv}(\mathrm{f} 1, \mathrm{x} ")$

$>\mathrm{x}<-2$

\# 生成导数公式

$>\operatorname{eval}(\mathrm{dx})$

\# 给自变量赋值

\# 计算导数值

运行上面的代码, 输出结果为 4.416147, 即所给函数在点 $\mathrm{x}=2$ 处的导数值。

实例 2. 求函数 $z=x^{2}+2 x y-y^{3}$ 在点 $(1,2)$ 处的偏导数

$>\mathrm{f} 2<-\operatorname{expression}\left(\mathrm{x}^{\wedge} 2+2^{*} \mathrm{x} * \mathrm{y}-\mathrm{y}^{\wedge} 3\right) \quad \#$ 定义函数

$>\mathrm{Dx}<-\mathrm{D}(\mathrm{f} 2, \mathrm{x} ") ; \mathrm{Dx}$

\# 生成函数关于自变量 $\mathrm{x}$ 的偏导函数

$>$ Dy <- D(f2, "y"); Dy

\# 生成函数关于自变量 $\mathrm{y}$ 的偏导函数

$>\mathrm{x}<-1 ; \mathrm{y}<-2$

\# 给自变量赋值

$>\operatorname{eval}(\mathrm{Dx}) ; \operatorname{eval}(\mathrm{Dy})$

\# 计算偏导数值

运行上面代码, 输出结果为 $6,-10$, 即为所给二元函数在点 $(1,2)$ 处的分别关于自变量 $\mathrm{x}$ 和 $\mathrm{y}$ 的 偏导数值。

4.4. 求定积分或广义积分

实例 1 . 求函数 $\mathrm{y}=\mathrm{x}^{2}$ 在 $[0,1]$ 上的定积分。

$>\mathrm{f} 3<-$ function $(\mathrm{x}) \mathrm{x}^{\wedge} 2$

\# 定义函数

$>$ integrate $(\mathrm{f} 3$, lower $=0$, upper $=1) \quad$ \# 计算积分

输出结果为: 0.3333333 with absolute error $<3.7 \mathrm{e}-15$.

实例 2 . 求函数 $y=1 /\left(1+\mathrm{x}^{2}\right)$ 在区间 $[-\infty,+\infty]$ 上的广义积分。

$>\mathrm{f} 4$ <- function $(\mathrm{x}) 1 /\left(1+\mathrm{x}^{\wedge} 2\right) \quad$ \# 定义函数

$>$ integrate(f4, lower=-Inf, upper=Inf) \# 计算积分

输出结果为: 3.141593 with absolute error $<5.2 \mathrm{e}-10$. 
4.5. 正态分布参数的几何意义

$\mathrm{R}$ 软件也可非常方便地运用于概率论教学。

实例 1 . 绘制不同参数的正态分布密度曲线图像。

$>\mathrm{x}<-\operatorname{seq}(-5,5$, length $=100)$

$>\operatorname{plot}(x, \operatorname{dnorm}(x, 0,1), x \lim =c(-5,5), y \lim =c(0,0.9)$, type="l", ylab="density", main="正态分布")

$>$ lines $(x, \operatorname{dnorm}(x, 0,0.5), 1$ ty $=2, \operatorname{col}=2)$

$>$ lines $(\mathrm{x}, \operatorname{dnorm}(\mathrm{x}, 0,2), 1 \mathrm{ty}=3, \mathrm{col}=3)$

$>\operatorname{lines}(\mathrm{x}, \operatorname{dnorm}(\mathrm{x},-2,1), \mathrm{lty}=4, \mathrm{col}=4)$

$>$ legend("topright", legend= paste("mu=",c(0,0,0,-2)," sd=", c(1,0.5,2,1)), lty=1:4, col=1:4)

上述 $\mathrm{R}$ 命令的输出见图 2 。

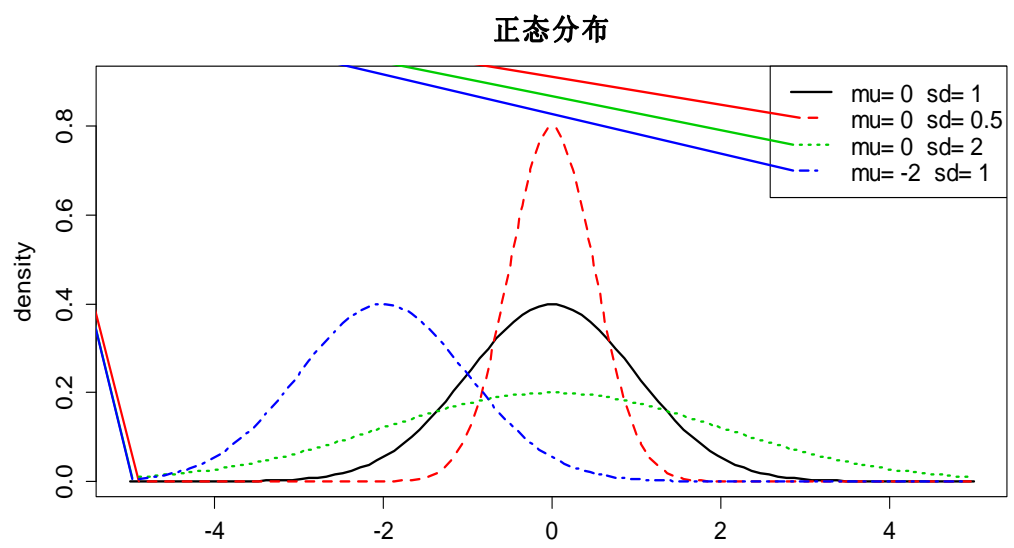

图 2

\section{5. 结论}

《医用高等数学》实验内容广泛, 包括函数图形演示、数值计算、数学建模、统计与模拟等。 数学实验不仅可以加深学生对数学知识的理解, 还可以使学生摆脱繁琐的计算, 进而激发学 生学习的兴趣, 增添学习的动力, 从而为更好的应用专业知识处理实际问题奠定基础。本文 结合实例, 介绍了 $\mathrm{R}$ 软件在数学实验教学中的具体应用, 从这些实例可以看出, $\mathrm{R}$ 软件能非 常高效地解决很多数学问题, 可以成为数学实践教学中的一个有力工具。相比其它软件, $\mathrm{R}$ 软件更擅长于统计计算, 目前国外很多高校的统计课都是结合 $\mathrm{R}$ 授课。 $\mathrm{R}$ 软件在我国的流行 虽是近几年的事, 但已经展现出潜力和活力。《医学统计学》是医学专业另一门重要的专业基 础课, 统计计算是医学统计实验课的重要组成部分。数学实验课中 $\mathrm{R}$ 软件的学习实践, 也为 学生随后学习《医学统计学》课程打下良好的基础。通过两门课程实验课软件工具的统一, 两门课程能够实现自然衔接, 大大节省学生学习时间, 提高教学效率。因此, 在医学数学乃 至统计学教学中采用 $\mathrm{R}$ 软件作为教学辅助工具, 值得推广。

\section{6. 致谢}

本文系湖北省教育科学 “十二五” 规划 2012 年度立项课题成果 (2012B240)。

\section{参考文献}

[1] 何兰, 滕辉, 宋运娜等. 医学院校高等数学课程教学改革探索与实践 $[\mathrm{J}]$. 西北医学教 育, $2015 ; 23(2)$ : 322-324.

[2] R: A language and environment for statistical computing. R Foundation for Statistical Computing, Vienna, Austria. URL http://www.r-project.org/. 
[3] 刘东海，彭丹. 基于 $\mathrm{R}$ 的概率统计实验教学模式探讨 $[\mathrm{J}]$. 当代教育理论与实践, 2014 , $6(7): 43-45$.

[4] 肖寅东, 刘科. $\mathrm{R}$ 统计软件在实验教学中的应用 $[\mathrm{J}]$. 实验科学与技术, 2013，11（2): 27-30.

[5] 武志辉，陈东彦，李冬梅. 以数学建模竞赛为平台辅助 $\mathrm{R}$ 软件教学 $[\mathrm{J}]$ 。高师理科学刊, 2015, 35 (5): 70-72.

[6] 程胜. $\mathrm{R}$ 统计软件及其在《时间序列分析》实践教学中的应用 $[\mathrm{J}]$. 教育教学论坛, 2014; 10 : $173-175$.

[7] 张选群. 医学高等数学 [M]. 北京: 人民卫生出版社, 2013.

[8]薛毅. R 语言实用教程 [M]. 北京: 清华大学出版社, 2014 .

\section{Acknowledgement}

This research was financially supported by the 2012 research Project of Higher Education Reform in Hubei Province (2012B240).

\section{References}

[1] Lan He, Hui Teng, Yunna Song. Exploration and Practice on teaching Reform of higher mathematics course in Medical Colleges [J]. Northwest Medical Education, 2015, 23(2): 322-324.

[2] R: A language and environment for statistical computing. $\mathrm{R}$ Foundation for Statistical Computing, Vienna, Austria. URL: http://www.r-project.org/.

[3] Donghai Liu, Dan Peng. On the experimental teaching mode of probability and statistics based on R software [J]. Theory and Practice of Contemporary Education, 2014, 6(7): 43-45.

[4] Yindong Xiao, Ke Liu. Application of R statistical software in experimental teaching [J]. Experiment Science and Technology, 2013, 11(2): 27-30.

[5] Zhihui Wu, Dongyan Chen, Dongmei Li. Using mathematical modeling contest as a platform to support the teaching of R software [J]. Journal of Science of Teachers' College and University, 2015, 35(5): 70-72.

[6] Shen Cheng. R statistical software and its application in the practice teaching of Time Series analysis [J]. Education Teaching Forum, 2014, 10: 173-175.

[7] Xuanqun Zhang. Medical Advanced Mathematics [M]. People's Medical Publishing House, 2013.

[8] Yi Xue. Practical tutorial for R language [M]. Tsinghua University Press, 2014.

第一作者简介: 赵军 (1978一), 男, 湖北十堰, 讲师, 主要研究方向: 应用统计学, E-mail: 427511@qq.com;

通讯作者简介: 杨琳 (1982-) , 女, 湖北省丹江口市, 讲师, 主要研究方向: 卫生法, E-mail: 5810030@qq.com。 